\title{
DVT prophylaxis in relation to patient risk profiling - the TUNE-IN study
}

\author{
P F Wessels, W J Riback
}

Background. Patients in acute hospital care show a high risk for venous thromboembolism (VTE); $52 \%$ of patients globally are at risk, with approximately only half receiving appropriate prophylaxis.

Objectives. The TUNE-IN study's primary objective was to evaluate the use of VTE prophylaxis in the private sector in South Africa in hospitalised patients in relation to patient risk profile, via both a clinical assessment and utilising a modified risk assessment model (RAM). The secondary objective was to evaluate the relationship between prophylaxis and the level of mobility at discharge as well as on days 3,7 and 14 post discharge.

Results. Of the 608 patients enrolled, $54.1 \%$ were evaluated to be at risk for VTE clinically whilst, with RAM assessment, $74.6 \%$ were assessed at risk. Adequate prophylaxis was given to only $70.9 \%$ of all patients.

Conclusion. Data appear to correlate with global findings (ENDORSE study): more than $50 \%$ of hospitalised patients are at risk for VTE. Clinical risk assessment does not correlate with RAM utilisation, resulting in possible over-diagnosis of VTE risk in lowand moderate-risk patients, with the converse happening in highand highest-risk patients. Certain risk groups are easily defined for VTE risk (e.g. major orthopaedic surgery), but individual risk assessment is crucial as it often reveals underlying and overlooked risk factors, with BMI and age the most frequently overlooked risk factors.

S Afr Med J 2012;102:85-89.
The ENDORSE study ${ }^{1}$ found that, globally, more than $50 \%$ of hospitalised patients are at risk of VTE, with adequate prophylaxis (according to the 7th American College of Chest Physicians (ACCP) guidelines) being given to approximately only $58 \%$ of surgical patients and $40 \%$ of medical patients.

The 8th ACCP guidelines ${ }^{2}$ were published in 2008, followed in 2009 by the South African guidelines. ${ }^{3}$ Despite consensus and approved guidelines, many patients at risk either do not receive prophylaxis, or receive inadequate prophylaxis. The TUNE-IN study was conducted to evaluate common practice in the assessment of VTE risk and concurrent prescription of prophylaxis in the private healthcare setting in South Africa. Perceived clinical VTE risk, actual VTE risk scoring (using an approved risk assessment model - modified Caprini risk assessment model), actual VTE prophylaxis prescribed, and mobilisation at varying post-discharge dates were collected.

\section{Methods \\ Settings and patients}

All patients were enrolled between May 2008 and November 2008 in a regional, prospective, cross-sectional and longitudinal, noninterventional, multi-site, observational study. Twenty consecutive patients, admitted for a minimum of one night, were evaluated at each of 29 sites across Gauteng by specialist physicians and surgeons. The sites were selected on the basis that they practised standard care in terms of VTE profiling and prophylaxis among private practitioners in Gauteng. As this condition might result in

Consultant haematologist, Ampath Laboratory, Pretoria; part-time consultant, Department of Medical Oncology, Steve Biko Hospital, Pretoria; clinical haematologist in private practice, Pretoria

P F Wessels, MB ChB, MMed (Haem), Cert Clin Haem (CMSA)

SANOFI South Africa, Johannesburg

W J Riback, MB BCh, DA (CMSA) bias, the selection may not reflect standard of care in all private and public hospitals, but is likely to be a fair representation of practice across the country.

\section{Data collection}

Participating sites, when introduced to the study, were asked to evaluate patients at risk for VTE clinically, without using any official scoring system. The patients were then re-evaluated via an approved risk assessment model (RAM) - modified Caprini risk score (Table I). This scoring system assigns different risk factors a specific point value. The total VTE risk is then calculated by adding the risk factors and the patient is then assigned to a risk category (low, moderate, high and highest groups) (Table II). The decision about VTE prophylaxis prescription was left to the treating physician/surgeon, but the recommended VTE prophylaxis for each group was supplied with the RAM. A bleeding risk reminder was also given with the risk assessment form (Table III). Specific recommendations given by the doctor for that specific patient were noted (Fig. 1).

No risk factor score for HIV disease was given; however, as it is a significant risk for VTE, it was noted as an additional comment.

Upon discharge, the patient's mobility and VTE prophylaxis were evaluated. Follow-up assessment of mobility was carried out telephonically on days 3, 7 and 14 (Fig. 1).

\section{Patient characteristics}

The study was planned to enroll 650 patients, with a minimum of 385 patients needed for statistical purposes; a total of 608 patients were enrolled, with the following exclusion criteria: $<18$ years old; already on anticoagulation therapy; and no signed patient informed and data release consent.

The 608 patients comprised 219 medical and 389 surgical patients; the male:female ratio was 49.8:50.2 (Table IV). Diagnoses were reported for 584 patients ( 365 surgical, 219 medical). The most common surgical patients were orthopaedic; respiratory infection was the most common medical condition resulting in admission. Surgical procedures were reported for all 389 surgical patients, with orthopaedic surgical procedures the largest subgroup. 
Table I: Modified Caprini risk assessment model

Each of the following risk factors represents 1 point: Age 41 - 59 years

Minor surgery planned

History of prior major surgery ( $<1$ month)

Varicose veins

History of inflammatory bowel disease

Swollen legs (current)

Obesity (BMI $>30 \mathrm{~kg} / \mathrm{m} 2)$

Acute myocardial infarction ( $<1$ month)

Congestive heart failure $(<1$ month)

Sepsis ( $<1$ month)

Serious lung disease incl. pneumonia ( $<1$ month)

Abnormal pulmonary function (COPD)

Medical patient currently at bed rest

Leg plaster cast or brace

Central venous access

Each of the following risk factors represents 2 points: Age 60 - 74 years

Major surgery ( $>60$ minutes)

Arthroscopic surgery ( $>60$ minutes)

Laparoscopic surgery ( $>60$ minutes)

Previous malignancy

Morbid obesity (BMI $>40 \mathrm{~kg} / \mathrm{m}^{2}$ )

Each of the following risk factors represents 3 points: Age $\geq 75$ years

Major surgery lasting 2 - 3 hours

BMI $>50 \mathrm{~kg} / \mathrm{m} 2$ (venous stasis syndrome)

History of SVT, DVT/PE

Family history of DVT/PE

Present cancer or chemotherapy

Congenital thrombophilia

Positive factor V Leiden

Positive prothrombin 20210A

Elevated serum homocysteine

Acquired thrombophilia:

Positive lupus anticoagulant

Elevated anticardiolipin antibodies

Heparin-induced thrombocytopaenia (HIT)

Other thrombophilia

Each of the following risk factors represents 5 points: Elective major lower extremity arthroplasty

Hip, pelvis or leg fracture ( $<1$ month)

Stroke $(<1$ month)

Multiple trauma ( $<1$ month)

Acute spinal cord injury (paralysis), ( $<1$ month)

Major surgery $>3$ hours

For women only (each risk factor represents 1 point):

Oral contraceptives or hormone replacement therapy

Pregnancy or postpartum ( $<1$ month)

History of unexplained stillborn infant, recurrent spontaneous abortion $(\geq 3)$, premature birth with toxaemia or growth-restricted infant

\section{Statistical analysis \\ Primary analysis}

Risk assessment was done using the modified Caprini RAM score. Relevant risk factors were added to obtain the total risk factor score (TRFS value). The sites then grouped patients into low, moderate, high and highest risk for developing VTE.

\section{Secondary analysis}

The level of mobility was measured using a three-level scale. The percentage of patients in each level was determined and this value was then correlated with their TRFS as per the RAM.

\section{Results and discussion}

Participating sites clinically evaluated $329(54.1 \%)$ of the total number of patients to be at risk for VTE.

Calculation of the TRFSs in the case report forms for the study were checked by the data management team of SANOFI. Minor data errors were found in the scores of 161 patients (108 surgical and 53 medical), which were subsequently adjusted. After adjustment, none of the patients' risk levels was classified as 'low'. Within the surgical groups, $30.3 \%$ of patients fell in the high-risk group, and $54 \%$ fell in the highest group. Medical patients were $32 \%$ in the high, and $38.4 \%$ of patients within the highest, group (Table V).

The TRFS groups were cross-correlated with the initial individual clinical assessments. The results showed that approximately $74.6 \%$ of all patients were in the high and highest risk groups, with $67.1 \%$ of medical and $74.6 \%$ of surgical patients at risk (Fig. 2). This high percentage among surgical patients may be explained by the high number of orthopaedic patients in the total sample. The same figure shows the clinical initial evaluation correlated with the RAM score.

Comparing patients in the high and highest risk categories (RAM) with clinical assessment reveals evidence that approximately $20 \%$ of patients were missed for VTE risk if not using a scoring system (Table VI). This finding highlights the possibility in South Africa that, by only performing subjective clinical assessment for VTE, the risk is run of having patients under-diagnosed for high and highest risk, so adding to the health burden of treating VTEs. Conversely, it raises the question of possible over-treating of patients by relying only on clinical assessment and possible overdiagnosis of low-risk patients.

Using the corrected TRFS values compared with clinical evaluation showed that assessing healthcare providers were most accurate in defining the highest-risk group of patients. Fig. 3 graphically shows results of the corrected total RAM score, clinical evaluation, correlation between clinical evaluation and RAM assessment, and prophylaxis prescribed.

Correlating clinical evaluation and high- and highest-risk patients and looking at prescribed prophylaxis revealed:

- Of the 389 surgical patients, 204 (52.4\%) had been clinically diagnosed as at risk for VTE. Using a modified RAM, 328 $(84.3 \%)$ were in the high- and highest-risk levels. Prophylaxis was prescribed for $296(76.1 \%)$ of the patients, and appropriate prophylaxis for $223(67.9 \%)$ out of 328 high-risk patients.

- Of the 219 medical patients, 125 (57.1\%) had been clinically diagnosed as at risk for VTE. Using a modified RAM; 154 (70.4\%) were in the high- and highest-risk levels. Prophylaxis was prescribed for $161(73.5 \%)$ of the patients, and appropriate prophylaxis for 119 out of 154 (77.2\%) of high-risk patients.

- Of all 608 patients, $329(54.1 \%)$ had been clinically diagnosed as at risk for VTE. Using a modified RAM, 482 (79.2\%) patients were in the high- and highest-risk levels. Prophylaxis was prescribed for 
Table II. Risk levels and recommendations according to risk level

\begin{tabular}{llll}
\hline Total risk factor score & Incidence of DVT & Risk level & Prophylaxis regimen \\
\hline $0-1$ & $<10 \%$ & Low & No specific measures, early ambulation \\
2 & $10-20 \%$ & Moderate & ES, IPC, LDUH or LMWH \\
$3-4$ & $20-40 \%$ & High & IPC, LDUH or LMWH \\
$\geq 5$ & $40-60 \%(1-5 \%$ mortality $)$ & Highest & Pharmacological; LDUH, LMWH, warfarin or Fxa \\
& & & inhibitor alone or in combination with ES or IPC
\end{tabular}

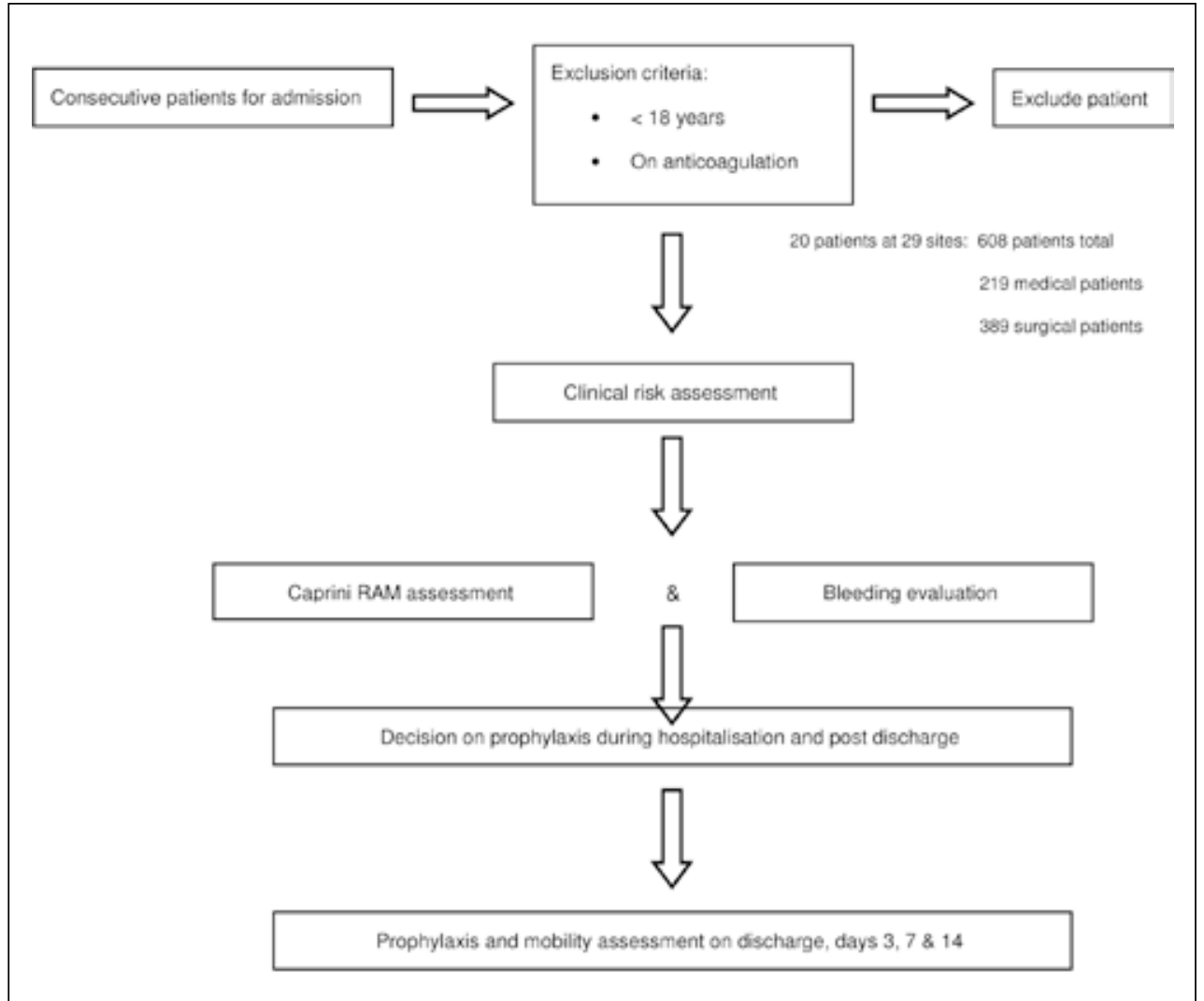

Fig. 1. Design of TUNE-IN study.

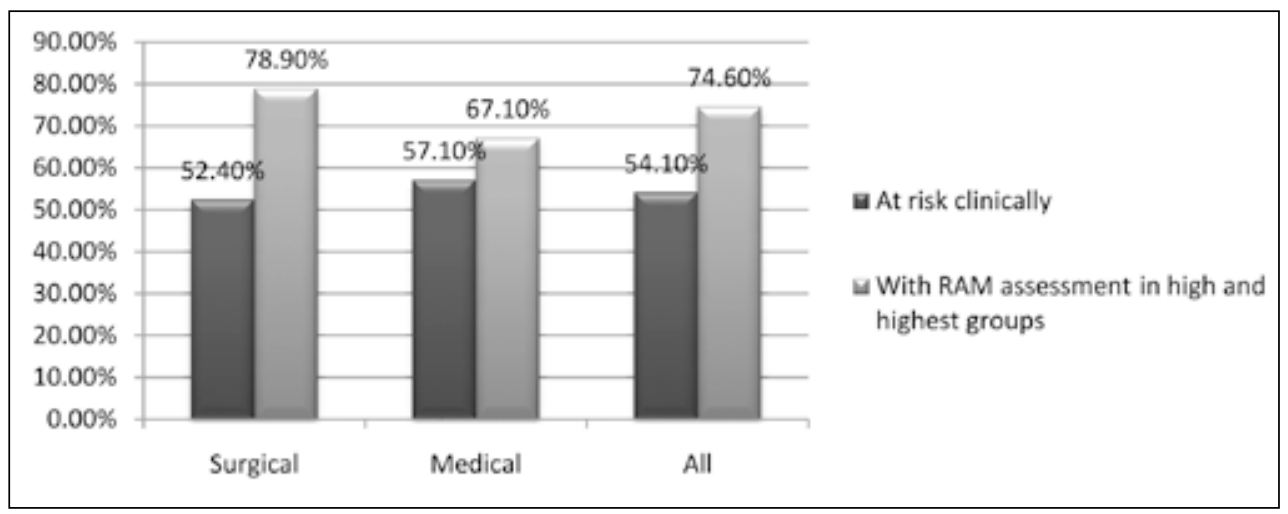

Fig. 2. Comparing clinical evaluation with TRFS patients at risk for VTE.

$457(75.2 \%)$ of the patients, and appropriate prophylaxis for 342 out of 482 (70.9\%) of high-risk patients.

Prophylaxis, in the context of this study, is important to define specifically whether it includes only early and aggressive mobilisation.
Treating sites recorded prophylaxis as all forms of prophylaxis, including early and aggressive mobilisation. In this paper, prophylaxis refers to all forms of prophylaxis, whereas 'appropriate prophylaxis' means more than just early and aggressive mobilisation, especially pertaining to the high- and highest-risk groups being compared with clinical risk evaluation.

\section{Reasons for not} prescribing prophylaxis

Reasons reported for not prescribing prophylaxis varied, with perception of low risk being the most common. Reasons why prophylaxis was not prescribed were reported for 151 patients (93 surgical, 58 medical). Bleeding risk as reason was given for $13.9 \%$ of patients. The high percentage (33.1\%) of patients who did not receive prophylaxis owing to early mobilisation could highlight the common misconception that early mobilisation is sufficient prophylaxis, even in high- and highest-risk patients.

\section{Prophylaxis prescribed}

The decision regarding prophylaxis was left to the treating physician or surgeon but, as indicated in Table III, the suggested prophylaxis for different RAM risk groups, as per the 8th ACCP and South African guidelines, was made available. To compare current acceptable prophylaxis regimens, only the high and highest RAM groups were evaluated for adequate prophylaxis.

Adequate prophylaxis according to RAM assessment guidelines was given in $223(67.9 \%)$ out of 328 surgical patients and $119(77.2 \%)$ 
Table III. Bleeding risk assessment

Has the patient, or a blood relative, ever received medical care for a bleeding tendency, e.g.:

- Nose bleeds or epistaxis?

- Excessive bleeding post dental procedure or extraction?

- Excessive bleeding after trauma or surgery?

- Excessive bleeding after tonsillectomy?

- Excessive bleeding during childbirth or during menstruation? (females only)

- Excessive bleeding from minor cuts?

Does the patient bruise easily?

Does the patient have a history of heparin-induced thrombocytopenia?

Is the patient's platelet count $<100000 / \mathrm{mm}^{3}$ or $>1000 / \mathrm{mm}^{3}$ ?

Is the patient taking aspirin or clopidogrel?

Does the patient have kidney or liver disease?

Is the patient on any medication (such as NSAIDS, and including natural/homoeopathic medication, e.g. garlic tablets, arnica, procydin) that increases the risk of bleeding?

Table IV. Patient demographics

\begin{tabular}{llll}
\hline Characteristic & $\begin{array}{l}\text { Surgical } \\
(N=389)\end{array}$ & $\begin{array}{l}\text { Medical } \\
(N=219)\end{array}$ & $\begin{array}{l}\text { All } \\
(N=608)\end{array}$ \\
\hline Age yrs/mean SD (range) & $49.7 / 17.6(18-99)$ & $52.6 / 17.9(20-96)$ & $50.7 / 17.8(18-99)$ \\
Male & $181(46.5 \%)$ & $122(55.7 \%)$ & $303(49.8 \%)$ \\
Female & $208(53.5 \%)$ & $97(44.3 \%)$ & $305(50.2 \%)$ \\
Weight kg/mean SD (range) & $83.8 / 20.6(40-185)$ & $79.9 / 22.7(40-200)$ & $82.4 / 21.5(40-200)$ \\
Height cm/mean SD (range) & $170.6 / 9.9(135-198)$ & $169.2 / 10.9(145-195)$ & $170.1 / 10.3(135-198)$ \\
\hline
\end{tabular}

Table V. Distributions of the adjusted TRFSs

\begin{tabular}{lllll}
\hline TRFS & Caprini VTE risk level & $\begin{array}{l}\text { Surgical } \\
(N=389)\end{array}$ & $\begin{array}{l}\text { Medical } \\
(N=219)\end{array}$ & $\begin{array}{l}\text { All } \\
(N=608)\end{array}$ \\
\hline $0-1$ & Low & $19(4.9 \%)$ & $31(14.1 \%)$ & $50(8.2 \%)$ \\
2 & Moderate & $42(10.8 \%)$ & $34(15.5 \%)$ & $76(12.5 \%)$ \\
$3-4$ & High & $118(30.3 \%)$ & $70(32.0 \%)$ & $188(30.9 \%)$ \\
$\geq 5$ & Highest & $210(54.0 \%)$ & $84(38.4 \%)$ & $294(48.4 \%)$ \\
\multicolumn{1}{|c|}{ Total } & & $389(100 \%)$ & $219(100 \%)$ & $608(100 \%)$ \\
\hline
\end{tabular}

out of 154 medical patients, being 342 (70.9\%) out of a total of 482 patients. If all patients that were evaluated to be at risk of bleeding were added into the high and highest RAM groups, the percentage adequate prophylaxis would be $70.1 \%$ in surgical patients, $82.6 \%$ in medical patients, and $74.1 \%$ in all patients. These percentages compare favourably with the current trends worldwide (ENDORSE study).

\section{Duration of prophylaxis}

The duration of the prophylaxis due to be prescribed to patients was reported initially, and subsequently adjusted at discharge, and days 3, 7 and 14 post discharge. Several variables could compound evaluating the period of prophylaxis by including the fact that different patients were hospitalised for different periods and that prophylaxis was not defined as excluding early and aggressive mobilisation. Clinical events would also have influenced the number of days on prophylaxis. These factors make the evaluation of data difficult, but final data that included all forms of prophylaxis were available for 442 patients (surgical 288; medical 154).

Pharmacological prophylaxis for 35 of the study patients (surgical 25; medical 10) was stopped during their hospital stay. The overall mean duration of post-discharge prophylaxis for surgical and medical patients ( 7.63 days v. 2.31 days) differs significantly. There was no difference in days of prophylaxis post surgery for different risk groups according to the modified Caprini RAM within the surgical and medical groups of patients respectively.

\section{Mobility}

Mobility was evaluated at discharge, and days 3, 7 and 14 post discharge. Level 1 indicates bed rest or sedentary patients; level 2 was the same as level 1 but with bathroom privileges and patients requiring assistance; and level 3 patients were those capable of physical activity beyond bed and bathroom. 
Table VI. Patient distribution per VTE risk level both clinically assessed and according to RAM.

\begin{tabular}{|c|c|c|c|c|c|c|c|}
\hline \multirow[b]{2}{*}{ TRFS } & \multirow{2}{*}{$\begin{array}{l}\text { Caprini VTE risk } \\
\text { level }\end{array}$} & \multicolumn{2}{|c|}{ Surgical $(N=389)$} & \multicolumn{2}{|c|}{ Medical $(N=219)$} & \multicolumn{2}{|c|}{ All $(N=608)$} \\
\hline & & RAM score & Clinical correlation & RAM score & Clinical correlation & RAM score & Clinical correlation \\
\hline $0-1$ & Low & 29 & 2 & 37 & 8 & 66 & 10 \\
\hline 2 & Moderate & 53 & 12 & 35 & 14 & 88 & 26 \\
\hline $3-4$ & High & 111 & 44 & 71 & 46 & 182 & 90 \\
\hline$\geq 5$ & Highest & 196 & 146 & 76 & 57 & 272 & 203 \\
\hline Total & & 389 & 204 & 219 & 125 & 608 & 329 \\
\hline
\end{tabular}

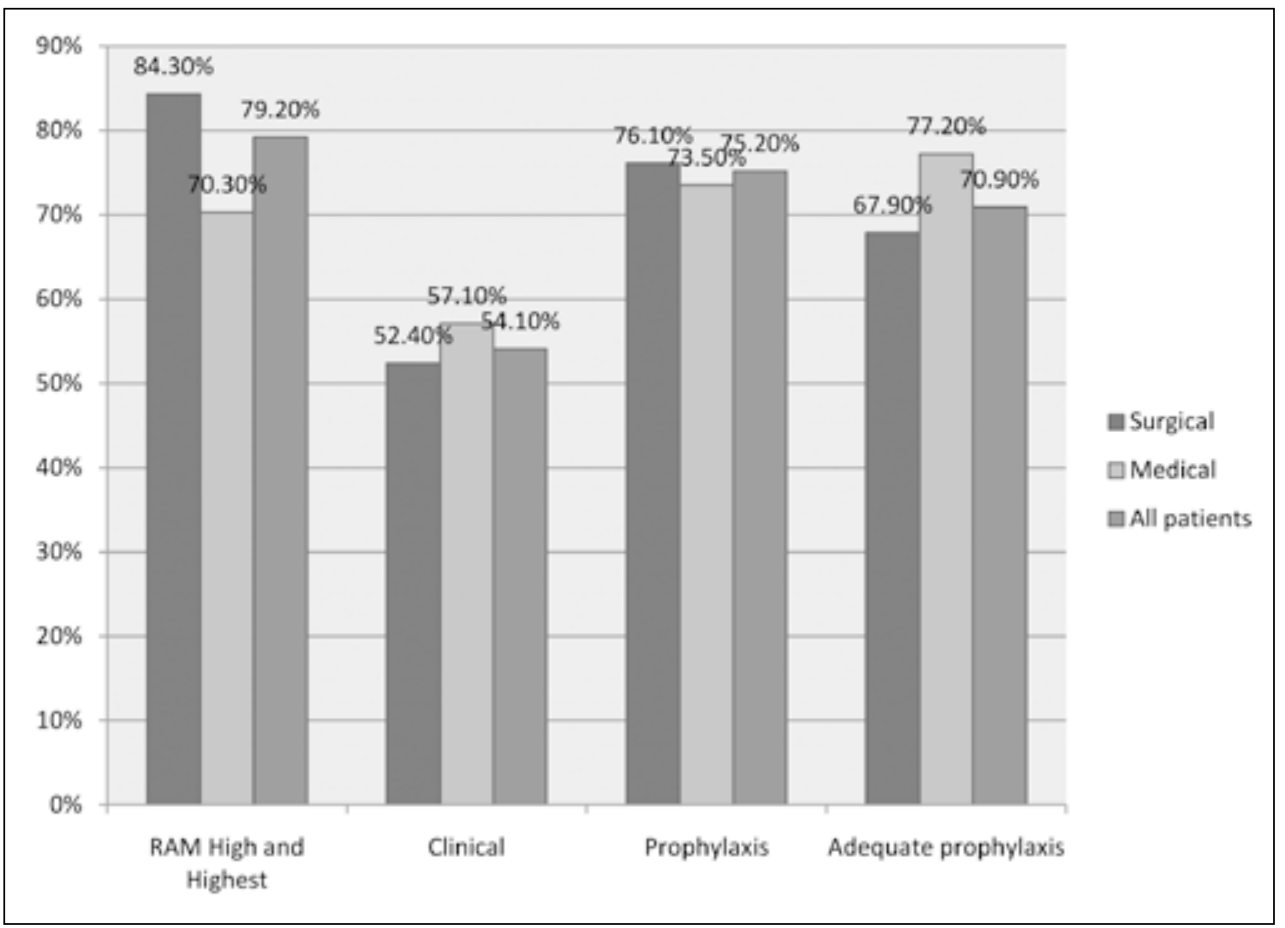

Fig. 3. Comparison between clinical evaluation, RAM high-risk patients and prophylaxis given.

Bleeding risk as reason for not prescribing prophylaxis is noted in a small percentage of patients (13.9\%). Some of the patients in the high- and very high-risk groups (which would correlate with the high-risk group in the ACCP 2008 guidelines for VTE prophylaxis) still only receive early and aggressive mobilisation (approximately $18 \%$ of surgical and $9 \%$ of medical patients, but bleeding risk might have influenced this result).

There is no correlation between length of prophylaxis post discharge and risk level of an individual patient. Although surgical patients are given prophylaxis for a longer period post discharge, medical patients show the same period of immobility post hospital discharge.

In South Africa, HIV disease is a major risk factor, and this

Medical and surgical patients showed a similar trend, with more than $90 \%$ of patients achieving level 3 mobility after 7 days. Conversely, at 14 days post discharge, there were 36 patients (surgical 21 , medical 15) who were not considered mobile, i.e. their mobility was at level 1 or level 2 .

\section{Conclusion}

This study used a small group of doctors in private practice, with possible bias in providing prophylaxis as a RAM scoring system was used v. clinical practice.

Data correlate with those of the ENDORSE study: more than $50 \%$ of hospitalised patients are at risk of developing thrombosis, and more than $70 \%$ of patients received adequate prophylaxis. This study also mirrors the AVAIL-ME study results presented at ISTH 2009, Boston, USA (which, by utilising ACCP guidelines, evaluated VTE prophylaxis prescribed with ACCP recommendations). ${ }^{4}$

The TUNE IN study noted that, if a structured RAM scoring system is used, a much higher percentage of patients are found to be at risk than that initially clinically evaluated. The two main factors that are underestimated are increased BMI and age. was omitted from the scoring system. Studies to evaluate the use of VTE across a wider spectrum of private and public sector patients should include this risk factor, which would add value to these findings.

Declarations. This study was funded by SANOFI South Africa (Pty) Ltd. Dr Wayne Riback is employed by SANOFI as Senior Medical Advisor. Data analysis was prepared and compiled by the Data Management Department of SANOFI, supported by independent data report via a contracted third party. Ethical approval for the study was obtained from Pharma Ethics and the relevant ethics committees in partaking private hospitals.

\section{References}

1. Cohen AT, Tapson VF, Bermann J-F, et al. Venous thromboembolism risk and prophylaxis in the acute hospital care setting (ENDORSE study): a multinational cross-sectional study. Lancet 2008;371:387-394. 2. Geerts WH, Bergqvist D, Pineo GF, et al. Prevention of venous thromboembolism: American College of Chest physicians evidence-based clinical practice guidelines (antithrombotic and thrombolytic therapy), 8th ed.: ACCP Guidelines. Chest 2008;133:381S-453S.

3. Jacobson BF, Louw $S$, Mer M, et al. Venous thromboembolism - prophylaxis and therapeutic practice guidelines. S Afr Med J 2009;99(6): 467-473.

4. Salameh P. AVAIL ME Project. ISTH meeting. Boston: 2009

Accepted 19 September 2011. 